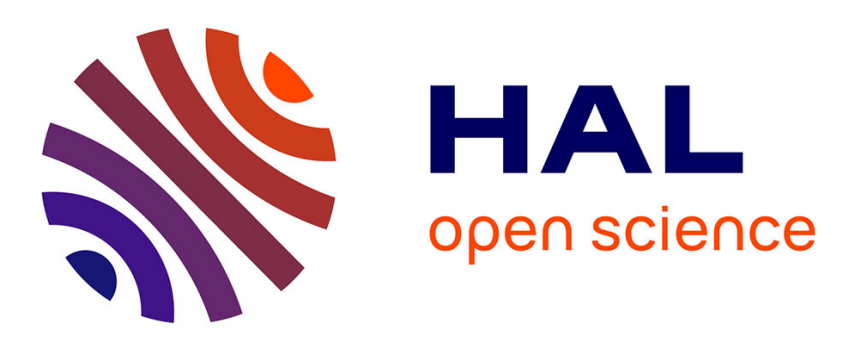

\title{
Les champs magnétiques continus et alternatifs dans l'espace proche et lointain de la terre
}

\author{
R. Gendrin
}

\section{To cite this version:}

R. Gendrin. Les champs magnétiques continus et alternatifs dans l'espace proche et lointain de la terre. Revue de Physique Appliquée, 1970, 5 (1), pp.209-220. 10.1051/rphysap:0197000501020900 . jpa-00243362

\section{HAL Id: jpa-00243362 https://hal.science/jpa-00243362}

Submitted on 1 Jan 1970

HAL is a multi-disciplinary open access archive for the deposit and dissemination of scientific research documents, whether they are published or not. The documents may come from teaching and research institutions in France or abroad, or from public or private research centers.
L'archive ouverte pluridisciplinaire HAL, est destinée au dépôt et à la diffusion de documents scientifiques de niveau recherche, publiés ou non, émanant des établissements d'enseignement et de recherche français ou étrangers, des laboratoires publics ou privés. 


\title{
LES GHAMPS MAGNÉTIQUES GONTINUS ET ALTERNATIFS DANS L'ESPAGE PROGHE ET LOINTAIN DE LA TERRE
}

\author{
Par R. GENDRIN, \\ Groupe de Recherches Ionosphériques (G.R.I.), Saint-Maur-des-Fossés.
}

\begin{abstract}
Résumé. - On rappelle les caractéristiques principales du champ magnétique terrestre et l'on indique pourquoi - à la surface de la Terre - l'augmentation de la précision des appareils de mesure en continu n'est pas d'une importance primordiale. Il n'en est pas de même pour l'espace interplanétaire dans lequel la précision absolue des mesures devrait être meilleure que $10^{-6}$ gauss.

L'intensité des fluctuations magnétiques naturelles décroissant très rapidement avec la fréquence, le problème de la sensibilité et de la bande passante des appareils de mesure se pose même dans l'espace proche, dans la gamme de fréquence de $0,1 \mathrm{~Hz}$ à $100 \mathrm{kHz}$. On rappelle les aspects principaux des phénomènes naturels se produisant dans cette bande de fréquence et l'on montre l'intérêt de leur observation.

Les techniques expérimentales adoptées sont décrites tant du point de vue de leur utilisation au sol qu'en fusée ou satellite.

Abstract. - We review the principal characteristics of the geomagnetic field of the Earth and we show that the sensitivity of the equipments is not the most important problem at the moment, at least for measuring continuous magnetic field at the surface of the Earth. On the contrary interplanetary measurements need sensitivities better than $10^{-6}$ gauss.

The mean spectral intensity of natural electromagnetic noise in the ULF $\left(10^{-2}-10 \mathrm{~Hz}\right)$ and the VLF $\left(10^{-1}-10 \mathrm{kHz}\right)$ ranges is rapidly decreasing with frequency. So that the problem of sensitivity and bandwith of the receiver system is a problem even in the near vicinity of the Earth. Principal aspects of these natural phenomena are reviewed, from the point of view of both their propagation characteristics and their generation mechanisms.

Technical aspects are also described and a special attention is paid to the problems which arise when measurements of low intensity magnetic fields (A.C. or D.C.) in space are needed.
\end{abstract}

Dans un colloque comme celui-ci, il ne saurait être question de faire une revue complète des phénomènes rencontrés en géophysique, dans le domaine qui nous occupe, encore moins d'en donner l'interprétation détaillée. Il importe de donner à l'ensemble des physiciens intéressés par la mesure des champs magnétiques faibles un aperçu sur les caractéristiques principales des phénomènes observés, afin qu'ils puissent se rendre compte des problèmes rencontrés et éventuellement proposer des solutions nouvelles que leurs études leur permettraient d'entrevoir.

Nous décrivons ici quelques-unes de ces caractéristiques, en nous limitant aux phénomènes naturels dont la fréquence est inférieure à quelques dizaines de kilohertz, et ce pour les raisons suivantes :

- Le plasma magnétosphérique est tel que, dans sa plus grande portion, la fréquence de plasma électronique $f_{0}$ est très supérieure à la gyrofréquence électronique $f_{B}$. Dans ces conditions, cette dernière joue, pour la plupart des ondes, le rôle d'une fréquence de coupure : ne se propagent que les ondes de fréquence $f<f_{B}$. A trois rayons terrestres et audelà, $f_{B}$ est lui-même inférieur à $30 \mathrm{kHz}$.

- Pour les fréquences supérieures à la dizaine de kilohertz, la détection des signaux fait appel à des techniques radioélectriques éprouvées, sensibles à la dérivée du champ $\mathrm{d} B / \mathrm{d} t$, plutôt qu'à des senseurs capables de mesurer la grandeur de $B$, même si cette grandeur est rapidement variable.

En fait, la détection des signaux magnétiques de fréquence supérieure à $10 \mathrm{~Hz}$ est actuellement toujours effectuée par des antennes à boucle ou à noyaux magnétiques (《 search coils »), les caractéristiques présentes des magnétomètres « continus » ne permettant pas d'étendre la bande passante au-delà de cette fréquence, en ayant une sensibilité suffisante. Néanmoins, une telle possibilité n'est pas à exclure dans un avenir proche, et comme, par ailleurs, les phénomènes électromagnétiques naturels présentent une certaine unité dans toute la gamme de 0 à quelques dizaines de kilohertz, nous avons préféré traiter le sujet dans son ensemble.

Données sur la dynamique des phénomènes étudiés. - La figure 1 donne une représentation schématique de l'intensité « moyenne » des signaux enregistrés au sol (pour les fréquences inférieures à $1 \mathrm{~Hz}$, on a donné les niveaux en $\left.\gamma^{(1}\right)$, en supposant un signal plus ou moins sinusoïdal; pour les signaux de fréquence supérieure à $1 \mathrm{~Hz}$, qui occupent une bande bien définie de fréquence, on a donné les niveaux en $\left.\gamma \cdot \mathrm{Hz}^{-1 / 2}\right)$. Sont également représentées trois bandes passantes caractéristiques moyennes d'appareils utilisés pour leur détection.

En ce qui concerne le domaine « continu », les variations observées peuvent aller de $20 \gamma$ (variation diurne à l'équateur) jusqu'à 500 ou $1000 \gamma$ (variations observées dans les régions aurorales au cours des

(1) $1 \gamma=10^{-5}$ gauss. 


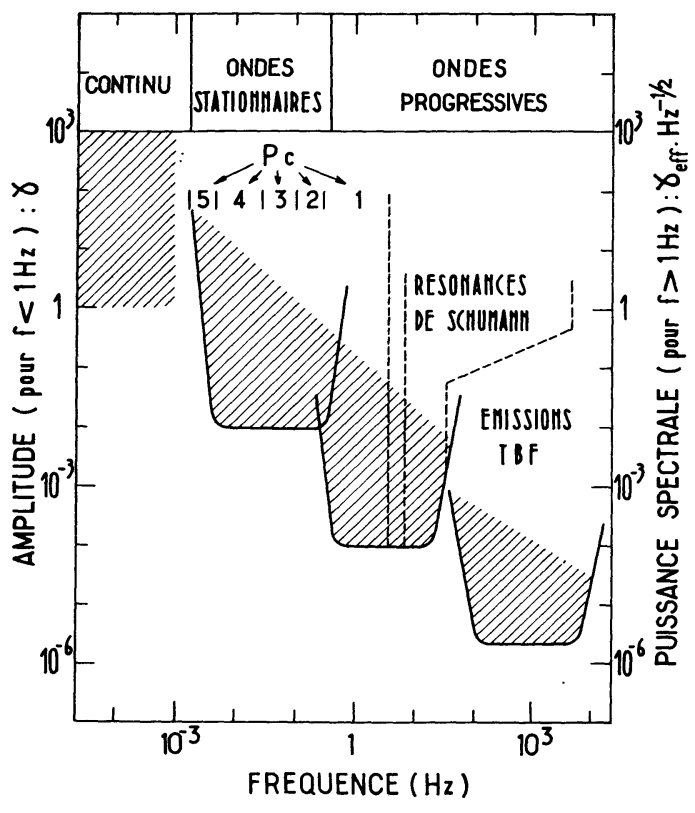

FIG. 1. - Niveau moyen des bruits U.B.F. et T.B.F enregistrés au sol.

orages ou sous-orages magnétosphériques). Les premières de ces variations sont assez bien connues, de sorte qu'une précision de l'ordre du $\gamma$ conserve un sens. La connaissance des champs magnétiques à la surface du globe avec cette précision permet de calculer les coefficients du développement en harmoniques sphériques, ce qui permet de représenter la valeur, en tout point de l'espace, du champ magnétique d'origine interne. Une précision supérieure serait sans utilité réelle, étant donné la présence de courants ionosphériques ou magnétosphériques intenses et variables.

Les résonances de Schumann. - Dans la bande de fréquence de 5 à $40 \mathrm{~Hz}$ apparaît un phénomène qui se distingue des autres par son origine et sa structure et que nous traiterons à part : il s'agit des résonances de la cavité Terre-Ionosphère ou résonances de Schumann.

L'extérieur de la Terre forme en effet une « cavité » au sens électromagnétique du terme, en ce sens que la solution des équations de Maxwell qui tient compte des conditions aux limites imposées à la surface d'une sphère conductrice conduit à l'existence d'une infinité de modes.

Un ordre de grandeur de la fréquence de résonance fondamentale est obtenu en divisant la vitesse de propagation de la lumière $(300000 \mathrm{~km} / \mathrm{s})$ par la circonférence de la Terre $(40000 \mathrm{~km})$. On aboutit ainsi à une fréquence de l'ordre de $8 \mathrm{~Hz}$ (fig. $2 \mathrm{a}$ ). En fait, le calcul exact fait intervenir les polynômes de Legendre et la fréquence théorique est légèrement différente: les fréquences des harmoniques ne sont pas des multiples entiers de la fréquence du fondamental. Par ailleurs, la présence de l'ionosphère, surface également conductrice quoique résistive, modifie les fréquences de résonances et introduit un élargissement des raies (fig. $2 \mathrm{~b}$ ). Le problème se complique par suite de la dissymétrie introduite entre l'hémisphère diurne et l'hémisphère nocturne (fig. $2 \mathrm{c}$ ).
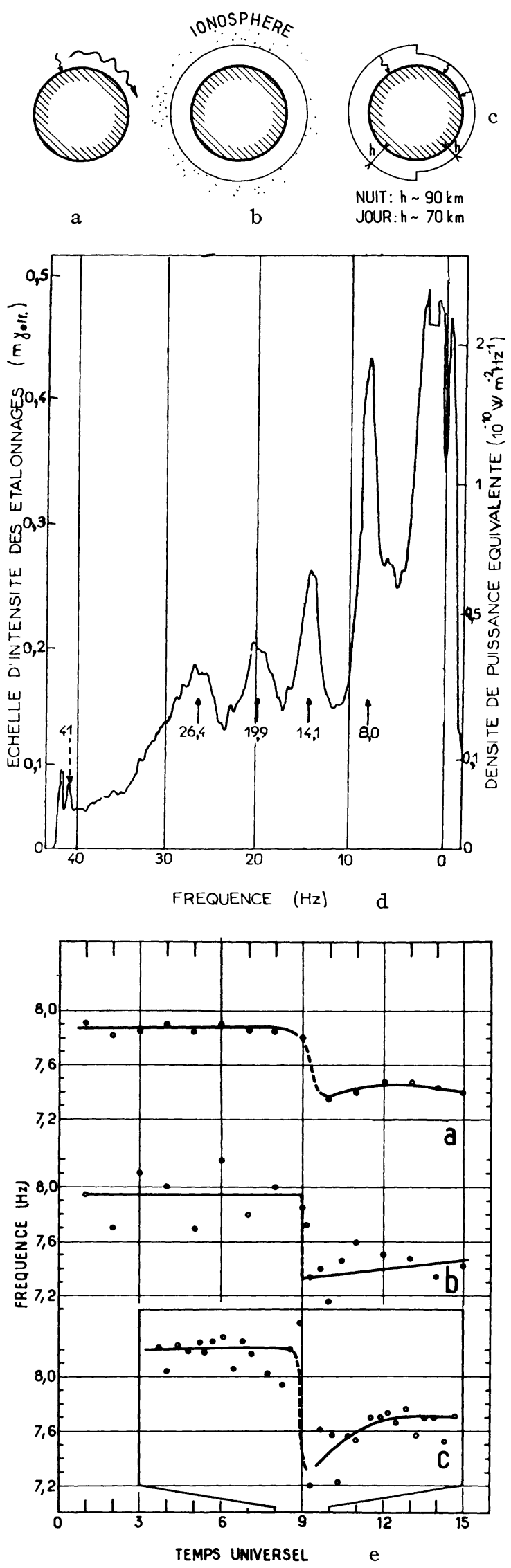

FIG. 2. - Résonances de la cavité Terre-Ionosphère : a), b), c) Origine.

d) Spectres (Gendrin et Stefant, C. R. Acad. Sci., 1962, 255, 2273).

e) Variations de fréquences (BENOIT et al., Ann. Geophys., 1967, 23, 132). 
L'observation expérimentale de ces phénomènes montre en général clairement l'existence des 3 ou 4 premiers harmoniques ( $f i g .2 \mathrm{~d}$ ). La fréquence du maximum du fondamental est sujette à des variations diurnes ou à des déplacements brutaux lorsqu'une perturbation affecte mondialement l'ionosphère (fig. $2 \mathrm{e}$ ), comme cela a été le cas lors de l'explosion thermonucléaire américaine à très haute altitude du 9 juillet 1962 .

Comme il s'agit d'un phénomène aléatoire (occurrence plus ou moins rapprochée des éclairs atmosphériques qui excitent ces résonances), la détermination de la forme du spectre de résonance ne se fera qu'après un temps d'intégration d'autant plus grand que l'on cherchera une précision meilleure.

Phénomènes d'origine magnétosphériques. Conditions de propagation. - Le plasma magnétosphérique est un milieu dont les paramètres caractéristiques locaux $\left(f_{0}\right.$ et $\left.f_{B}\right)$ varient dans des proportions énormes; ceci à cause de la décroissance rapide avec la distance géocentrique de la densité d'ionisation et de l'intensité du champ magnétique. Comme les ondes se propagent, ainsi qu'il sera montré plus loin, en suivant plus ou moins les lignes de force du champ magnétique terrestre, ce sont les valeurs de ces paramètres caractéristiques au sommet de la ligne de force qui jouent un rôle fondamental dans la définition et la propagation des ondes.

A titre d'exemple, considérons une ligne de force dont le point le plus éloigné est situé à une distance géocentrique de 5 rayons terrestres. La valeur du champ magnétique y est de l'ordre de $2500 \gamma$, la densité électronique de l'ordre de $40 \mathrm{~cm}^{-3}$; de sorte que :

$$
\begin{aligned}
& f_{0} \sim 60 \mathrm{kHz} \\
& f_{B} \sim 7 \mathrm{kHz} \\
& F_{B} \sim 4 \mathrm{~Hz}
\end{aligned}
$$

On utilise aussi les grandeurs :

$$
\begin{array}{ll}
\alpha=f_{0} / f_{B} & \text { caractéristique du milieu } \\
x=f \mid f_{B} & \text { caractéristique de la fréquence }
\end{array}
$$
et le rapport :

$$
m=M_{i} \mid M_{e} \sim 1840
$$

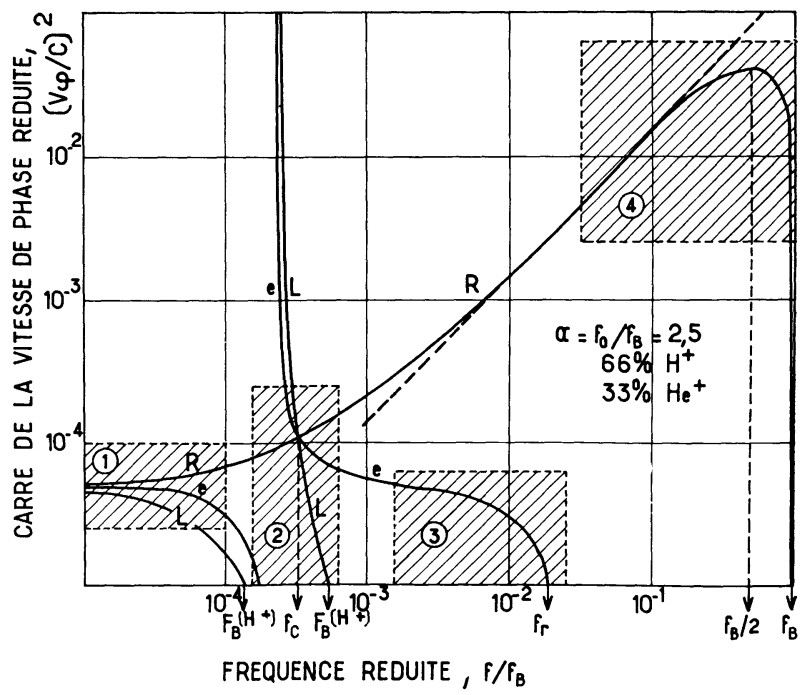

a entre la masse du proton et celle de l'électron; pour les valeurs indiquées, $\alpha$ est de l'ordre de 9 .

Les phénomènes de très basse fréquence (T.B.F.) sont alors définis comme ceux dont la fréquence est inférieure à la gyrofréquence électronique $f_{B}$; les phénomènes d'ultra-basse fréquence, comme ceux dont la fréquence est inférieure à la gyrofréquence protonique $F_{B}$.

La figure 3 a donne, pour des paramètres caractéristiques de valeur assez voisine de celles que nous venons d'énoncer, la variation de la vitesse de phase des ondes en fonction de la fréquence. Laissons de côté le fait que le milieu contient, sur cette figure, deux espèces d'ions positifs $\left(\mathrm{H}^{+}\right.$et $\left.\mathrm{He}^{+}\right)$, et ne nous occupons que des ondes en propagation longitudinale, pour lesquelles il existe deux modes, l'un polarisé à droite ( $R$ wave) et qui présente une fréquence de résonance

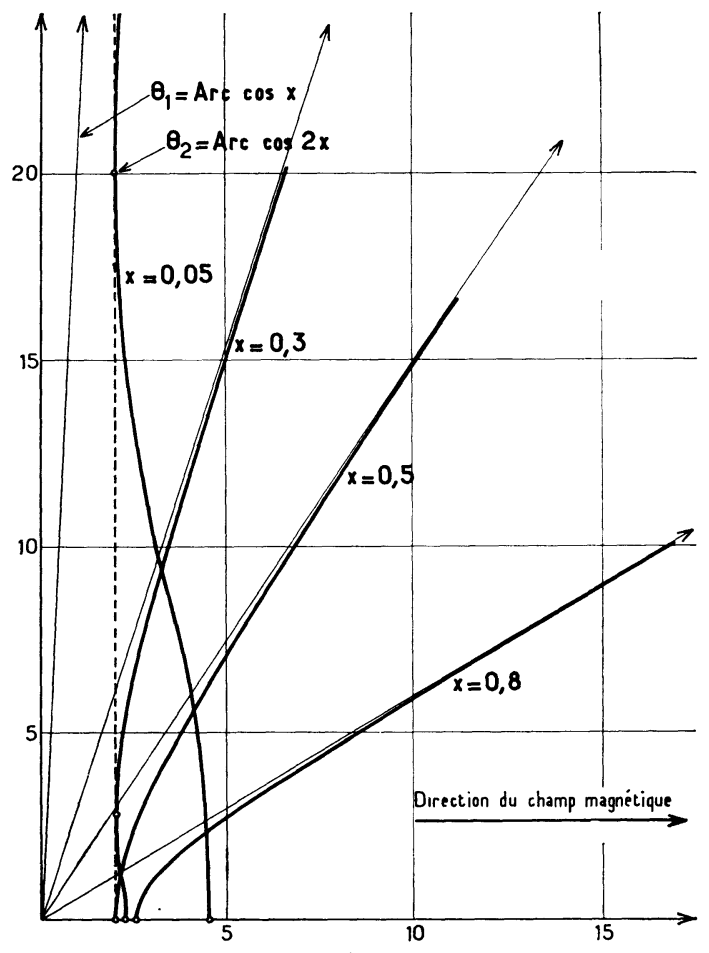

b

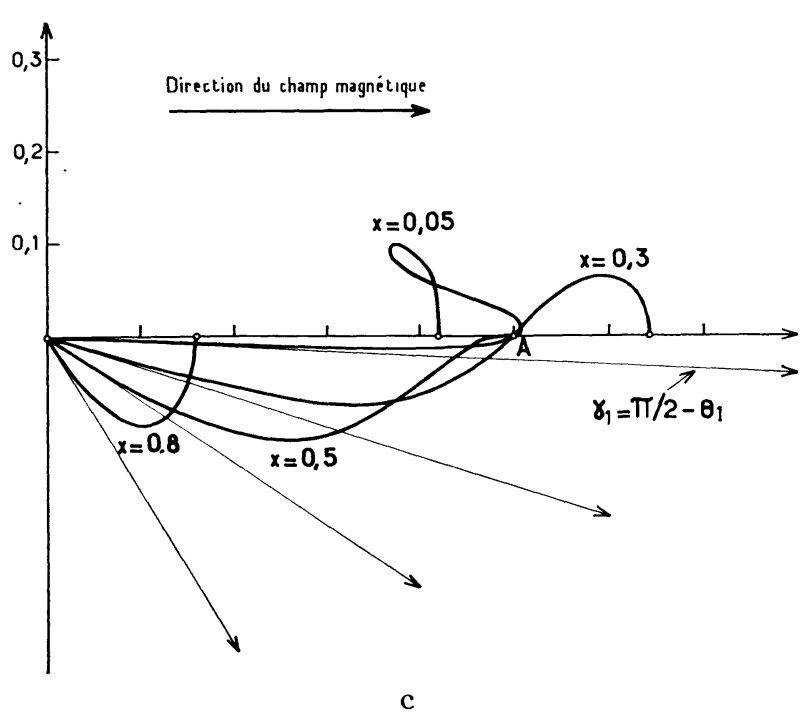

c

FIG. 3. - Caractéristiques de propagation (d'après GENDRIN, Handbuch der Physik, 1970) : a) $F_{B}\left(\mathrm{He}^{+}\right) F_{B}\left(\mathrm{H}^{+}\right)$; b) Indice normalisé $n / \alpha$; c) Vitesse de rayon normalisé $V \alpha / c$. 
pour $f=f_{B}$ : c'est le mode des « sifflements » radioélectriques et des principales émissions T.B.F. observées; l'autre, polarisé à gauche ( $\mathrm{L}$ wave), qui ne se propage que si $f<F_{B}$ : c'est le mode des émissions hydromagnétiques ou ondes U.B.F.

La valeur de la vitesse de phase de ces ondes peut être représentée dans un domaine restreint de fréquence par les expressions :

$v_{\varphi}=V_{a}=\frac{c}{\alpha \sqrt{m}}$ pour les ondes U.B.F.

$v_{\varphi}=\frac{c}{n_{\varphi}}=\frac{c \sqrt{x(1-x)}}{\alpha} \quad$ pour les ondes T.B.F.

On voit donc que les ondes d'ultra-basse fréquence se propagent sans dispersion à la vitesse de Alfven, tandis que les ondes de très basse fréquence se propagent avec une dispersion notable et avec des vitesses de phase environ 40 fois $(\sqrt{m})$ plus grandes.

A ces effets, réfringent et dispersif, il convient d'ajouter un effet d'anisotropie très important. L'indice de phase dépend de l'orientation de la normale d'onde par rapport au champ magnétique directeur, et par conséquent la direction de propagation de l'énergie diffère de la direction du vecteur d'onde. Cet effet a pour conséquence de limiter la propagation de l'énergie dans un cône de directions voisines du champ magnétique, d'où la propagation le long des lignes de forces. Courbes d'indice et courbes de vitesse du rayon sont représentées sur les figures $3 \mathrm{~b}$ et $3 \mathrm{c}$, pour des ondes T.B.F. Des courbes similaires peuvent être tracées dans le domaine U.B.F.; le « guidage » par le champ magnétique est encore plus important, au moins pour le mode polarisé à gauche (aux fréquences ultra-basses, le mode polarisé à droite est en effet quasi isotrope).

Ondes stationnaires, ondes progressives. - Ainsi qu'il a été dit au paragraphe précédent, les paramètres caractéristiques du milieu varient dans des proportions très importantes le long d'une même ligne de force. Néanmoins la valeur de ces paramètres au sommet de la ligne de force va nous permettre de fixer, au moins de façon qualitative, les ordres de grandeur mis en jeu dans les différents phénomènes (fig. 4).

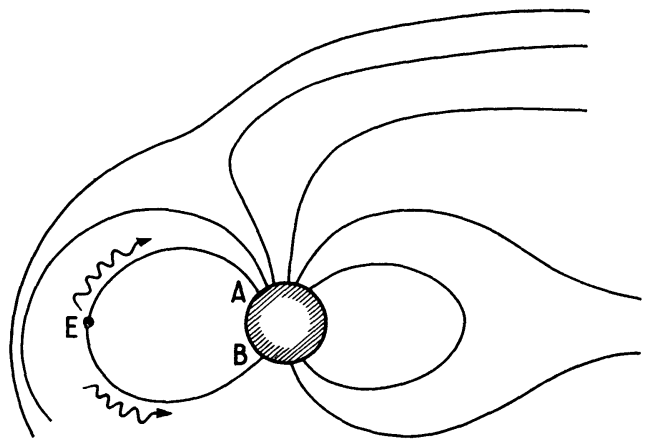

FIG. 4. - Propagation des ondes U.B.F. et T.B.F dans la magnétosphère : $V_{a}(E) \sim 1000 \mathrm{~km} / \mathrm{s}$; $L_{\mathrm{AEB}} \sim 70000 \mathrm{~km} ; f=0,02 \mathrm{~Hz} ; n \sim 300 ; \lambda=\mathrm{L}$; $f=1 \mathrm{~Hz} ; n \sim 300 ; \mathrm{L} \approx 70 \lambda ; T_{\mathrm{AB}} \sim 70 \mathrm{~s}$; $f=3 \mathrm{kHz} ; n_{\varphi} \sim 20 ; \lambda \sim 10 \mathrm{~km} ; n_{g} \sim 10 ; T_{\mathrm{AB}} \sim 5 \mathrm{~s}$.

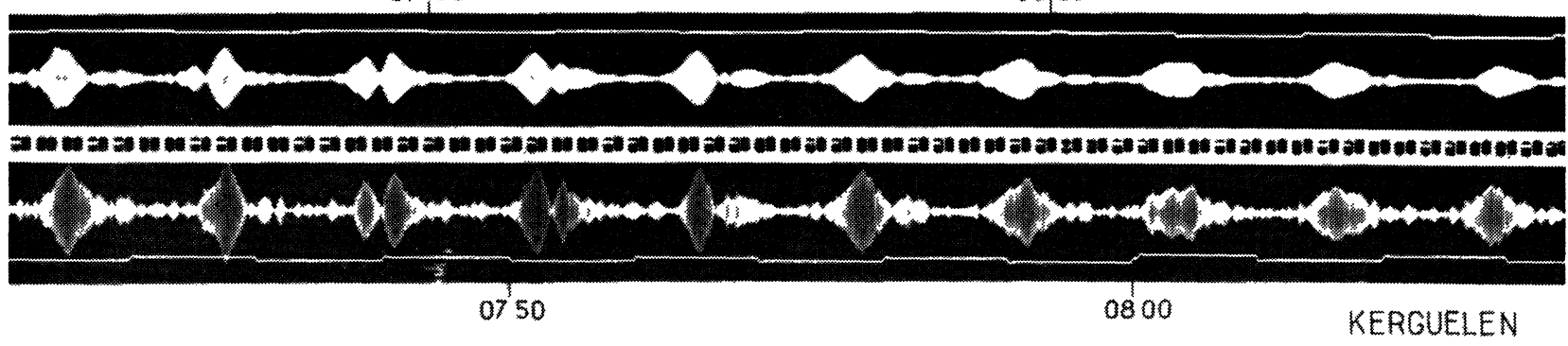

a

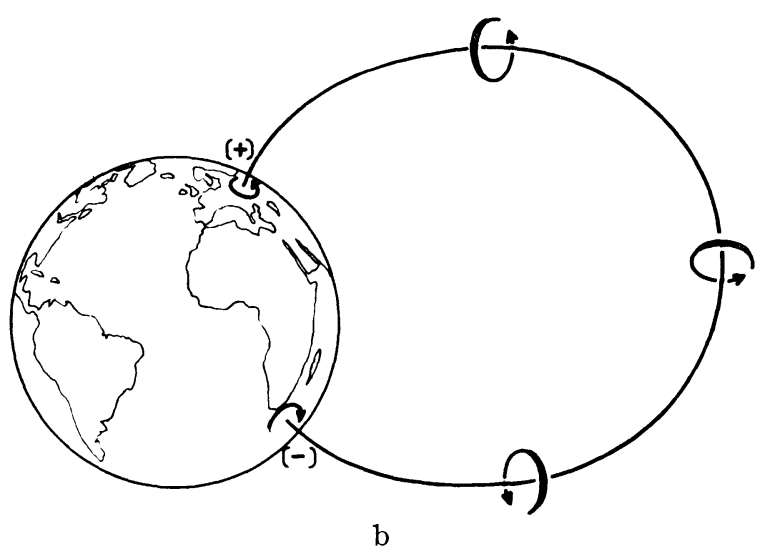

FIG. 5. - Propagation interhémisphère des oscillations " en perles ":

a) Temps (Gendrin, Thèse, 1967).

b) Polarisation (GENdRIN et al., Ann. Geophys., 1967, 22, 329). 
Avec les valeurs fournies par les équations (1) et (2), on voit que $V_{a}$ est de l'ordre de $1000 \mathrm{~km} / \mathrm{s}$ $\left(n_{\varphi}=n_{g} \approx 300\right)$ et que l'indice de phase pour une onde T.B.F. de fréquence voisine $3,5 \mathrm{kHz}$ est de l'ordre de 20. A cette fréquence, l'indice de groupe est approximativement la moitié de l'indice de phase. Avec ces valeurs, il est possible de calculer longueurs d'onde et temps de parcours pour différentes fréquences.

La longueur $\mathrm{L}$ de la ligne de force étant, dans l'exemple choisi, de l'ordre de $70000 \mathrm{~km}$, on voit que les pulsations continues de longue période ( $P c$ 2-3-4 de la fig. 1) ne peuvent être considérées comme des ondes qui se propagent. Il s'agit plutôt d'ondes stationnaires qui font intervenir des oscillations de l'ensemble de la cavité magnétcsphérique.
La valeur élevée de l'indice et les grandes distances mises en jeu permettent en revanche de considérer les phénomènes de fréquence aussi basse que $1 \mathrm{~Hz}$ comme des ondes qui se propagent. Le temps de propagation d'un hémisphère à l'autre est de l'ordre de la minute $(70000 \mathrm{~km} / 1000 \mathrm{~km} / \mathrm{s}=70 \mathrm{~s})$.

Aux fréquences plus élevées ( 1 à $5 \mathrm{kHz})$, la longueur d'onde est encore plus courte, bien que l'indice ait diminué. Les temps de parcours sont de l'ordre de 2 à 5 s.

Quelques évidences expérimentales de cette propagation d'un hémisphère à l'autre sont rassemblées sur les figures 5 et 6 :

Figure 5 a. - Oscillations « en perles», de fréquence voisine de $1 \mathrm{~Hz}$, enregistrée, en deux stations géomagnétiquement conjuguées (Kerguelen et Sogra).

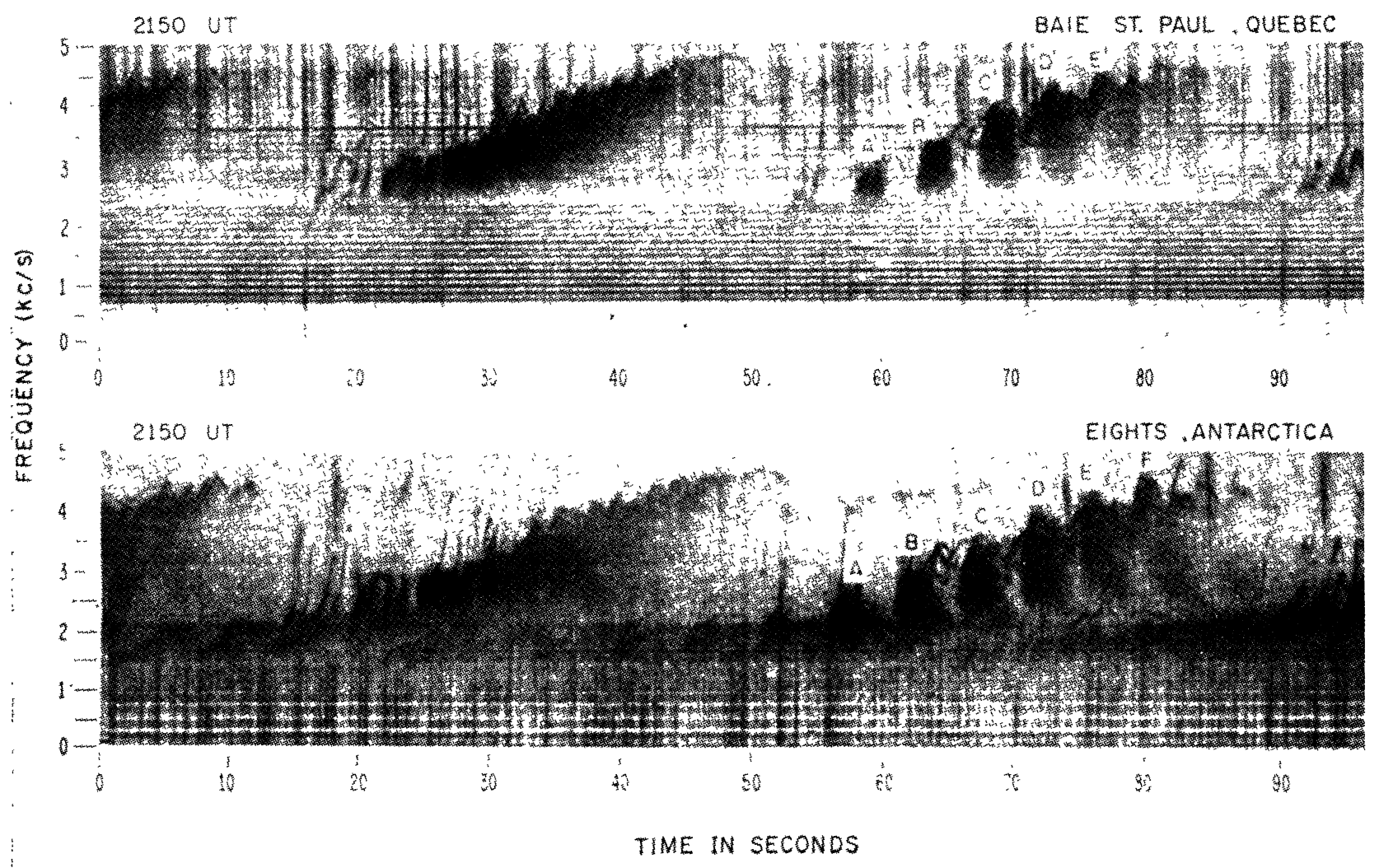

a

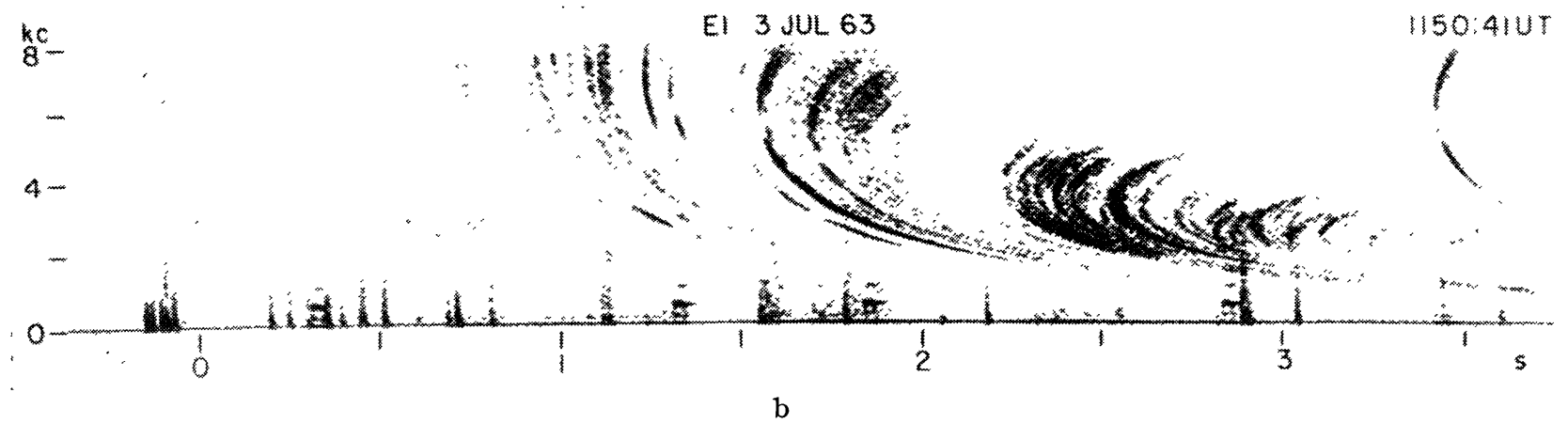

Fig. 6. - Preuves de la propagation des émissions T.B.F. et des sifflements radioélectriques :

a) Décalage en temps (CARson et al., J.G.R., 1965, 70, 4293).

b) Dispersion (CARPENTIER et SMITH, Rev. Geophys., 1964, 2, 415). 
On remarque la périodicité des phénomènes et leur correspondance un par un, à condition de déplacer l'axe des temps d'une station d'une quantité approximativement égale à la moitié de la période de répétition (voisine ici de $2^{\prime} 30^{\prime \prime}$ ).

Figure 5 b. - Toujours dans le domaine U.B.F., la possibilité de détecter la polarisation des signaux aux deux extrémités de la même ligne de force permet de montrer que la propagation a bien lieu dans un mode de polarisation déterminée (ici, le mode polarisé à gauche, dans le sens de rotation des protons autour du champ magnétique terrestre).

Les deux figures suivantes font appel à la représentation du type " sonagramme », où le noircissement est proportionnel à l'intensité du signal à un instant donné (en abscisse) et à la fréquence considérée (en ordonnée). Il s'agit en fait d'une analyse spectrale continue.

Figure 6 a. - Deux émissions T.B.F. sont reçues en deux points approximativement conjugués. Les différents éléments d'une même émission périodique $\mathrm{AA}^{\prime}, \mathrm{BB}^{\prime}, \ldots$, se correspondent deux à deux; le décalage en temps est de l'ordre de 3 s entre les deux stations.

Figure 6 b. - La théorie (formule (2)) prévoit une différence entre les temps d'arrivée des différentes fréquences. Il s'agit ici d'un sifflement produit par un éclair atmosphérique dont une partie de l'énergie radioélectrique de basse fréquence s'est propagée le long de plusieurs lignes de force (d'où les traces différentes). On remarque sur chaque trace la présence d'une fréquence qui se propage plus rapidement que les autres; c'est la fréquence de «nez » (« nose frequency ») dont l'origine est liée à la forme de la courbe de dispersion dans le mode droit (voir fig. 3 a).

Remarques sur la nature électromagnétique des ondes. - Arrivés à ce stade de notre exposé, il est important de se rendre compte des raisons qui font que c'est principalement l'aspect magnétique des phénomènes qui nous intéresse.

A l'intérieur de la plasmasphère $\left(R<5-6 R_{T}\right)$, la température du plasma est faible, disons de l'ordre de $1 \mathrm{eV}$, ce qui correspond à des vitesses thermiques de l'ordre de $600 \mathrm{~km} / \mathrm{s}$ pour les électrons et de $15 \mathrm{~km} / \mathrm{s}$ pour les protons. Dans ces conditions, le plasma peut être considéré comme froid, tant que la vitesse de phase des ondes reste grande par rapport à la vitesse thermique des particules qui interviennent dans la propagation (électrons pour les ondes T.B.F., protons pour les U.B.F.). Gette condition revient à dire que les effets thermiques peuvent être négligés tant que l'indice de phase défini par les équations (2) est inférieur à :

$$
\begin{aligned}
& 300000 / 15 \sim 20000 \text { pour les ondes U.B.F. } \\
& 300000 / 600 \sim 500 \text { pour les ondes T.B.F. }
\end{aligned}
$$

Ces conditions sont pratiquement toujours vérifiées (sauf au voisinage des résonances), on a donc affaire à des ondes électromagnétiques pures. Il n'en est plus de même dans les régions externes de la magnétosphère ou de la magnétogaine, régions dans lesquelles la température est nettement plus élevée (et la valeur 'du paramètre $\alpha=f_{0} / f_{B}$ nettement plus grande).

La raison pour laquelle tous les appareils détecteurs utilisés dans cette gamme de fréquence, aussi bien au sol qu'en satellite, sont des détecteurs magnétiques est la suivante : l'équation

$$
\operatorname{rot} E=-\partial B / \partial t
$$

montre que :

$$
|E|=\frac{\omega}{k}|B|=\frac{c|B|}{n_{\varphi}} .
$$

Par suite de la forte valeur de l'indice, c'est la composante magnétique de l'onde qui joue un rôle important et non pas sa composante électrique. Ceci n'est vrai qu'en propagation parallèle au champ magnétique; dans les autres cas, on n'a pas le droit de passer de (3) à (4). Les ondes à forte composante électrique - qui sont d'ailleurs observées dans la magnétosphère, mais ne peuvent parvenir jusqu'au sol - existent principalement dans les cas de propagation transverse.

Par ailleurs, dans le cas des résonances de Schumann, pour lesquelles les ondes se propagent à la vitesse de la lumière $\left(n_{\varphi}=1\right)$, on peut aussi bien détecter $E$ que $B$. Les deux types d'antennes ont d'ailleurs été utilisés.

Emissions naturelles. - Nous avons vu l'importance de détecter des signaux d'amplitude faible. Nous voulons montrer ici que la structure spectrale des phénomènes oblige à enregistrer ceux-ci dans des bandes larges.

Sur la figure 7 sont représentés sous forme de sonagramme les spectres de différents types d'émissions U.B.F.; les échelles de temps sont de l'ordre de l'heure et celles de fréquence de l'ordre du hertz. Sur la figure 8, sont représentés quelques types d'émissions T.B.F. Les échelles correspondantes sont de l'ordre de la seconde et du kilohertz.

On remarque l'existence, dans les deux bandes de fréquence, de deux types de phénomènes : les émissions discrètes (une fréquence émise qui varie rapidement avec le temps) et les émissions à bande large (où tout un spectre de fréquence est émis simultanément, dont les caractéristiques varient au cours du temps).

Le problème de l'origine de ces phénomènes est délicat et non encore résolu de façon satisfaisante. Disons simplement ici que leur interprétation fait appel à des mécanismes d'interaction entre les ondes qui peuvent se propager dans la magnétosphère et les particules énergétiques que l'on sait y exister en permanence.

Les ondes, une fois engendrées, réagissent à leur tour sur les particules et modifient leur distribution angulaire ou énergétique. Il en résulte une accélération ou une décélération des particules, un piégeage plus accentué (élévation des points miroirs) ou une précipitation accrue. On conçoit donc que l'étude détaillée de ces phénomènes soit d'un très grand intérêt pour la compréhension de la dynamique de la magnétosphère. 

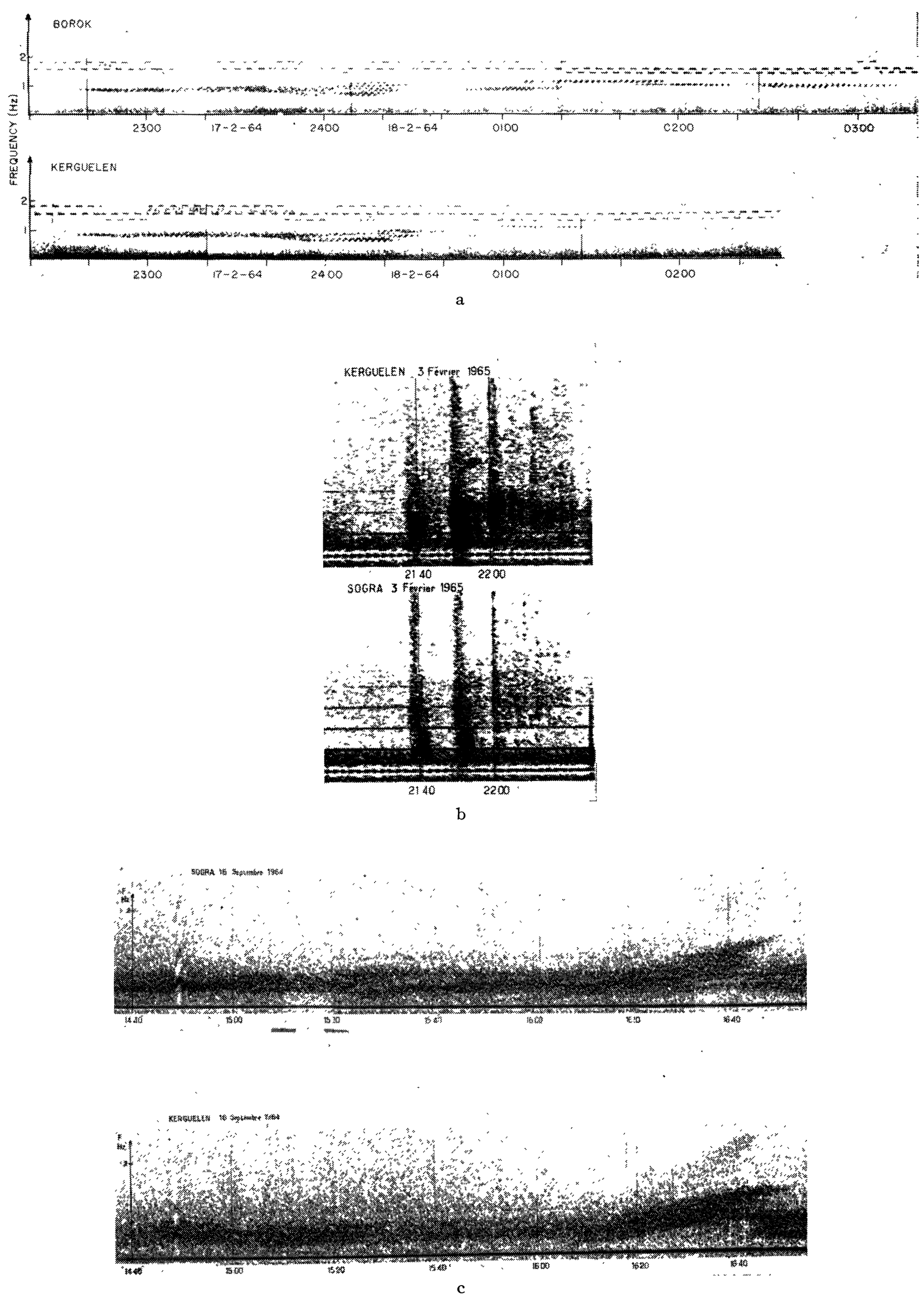

FIG. 7. - Différents types d'émissions U.B.F. :

a) Pulsations "en perles "(GEndrin et Troitskaya, Radio Sci., 1965, 69 D, 1107).

b) Pulsations irrégulières courtes (GENDRIN et LACOURLY, Ann. Geophys., 1968, 24, 267).

c) Pulsations irrégulières de périodes décroissantes (GENDRIN et al., Planet. Space Sci., 1! 67, 15, 1239). 

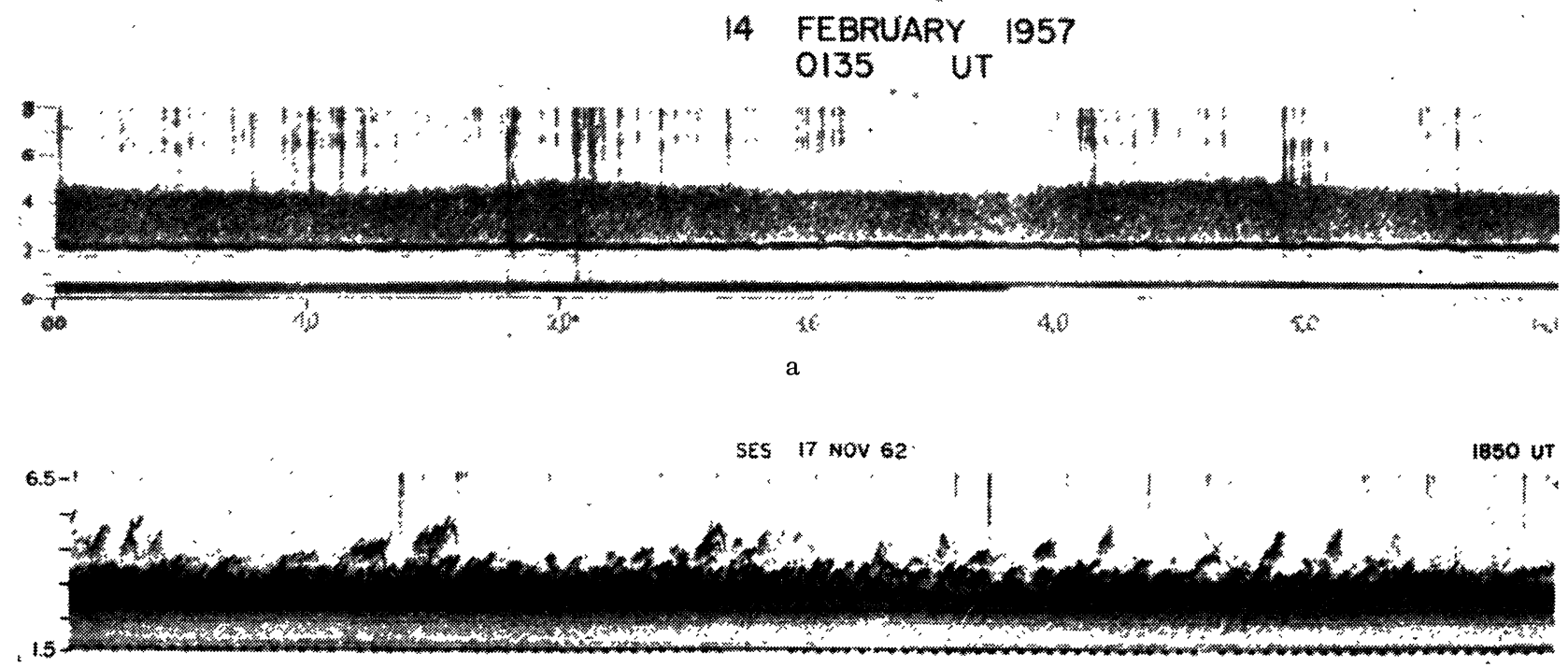

b
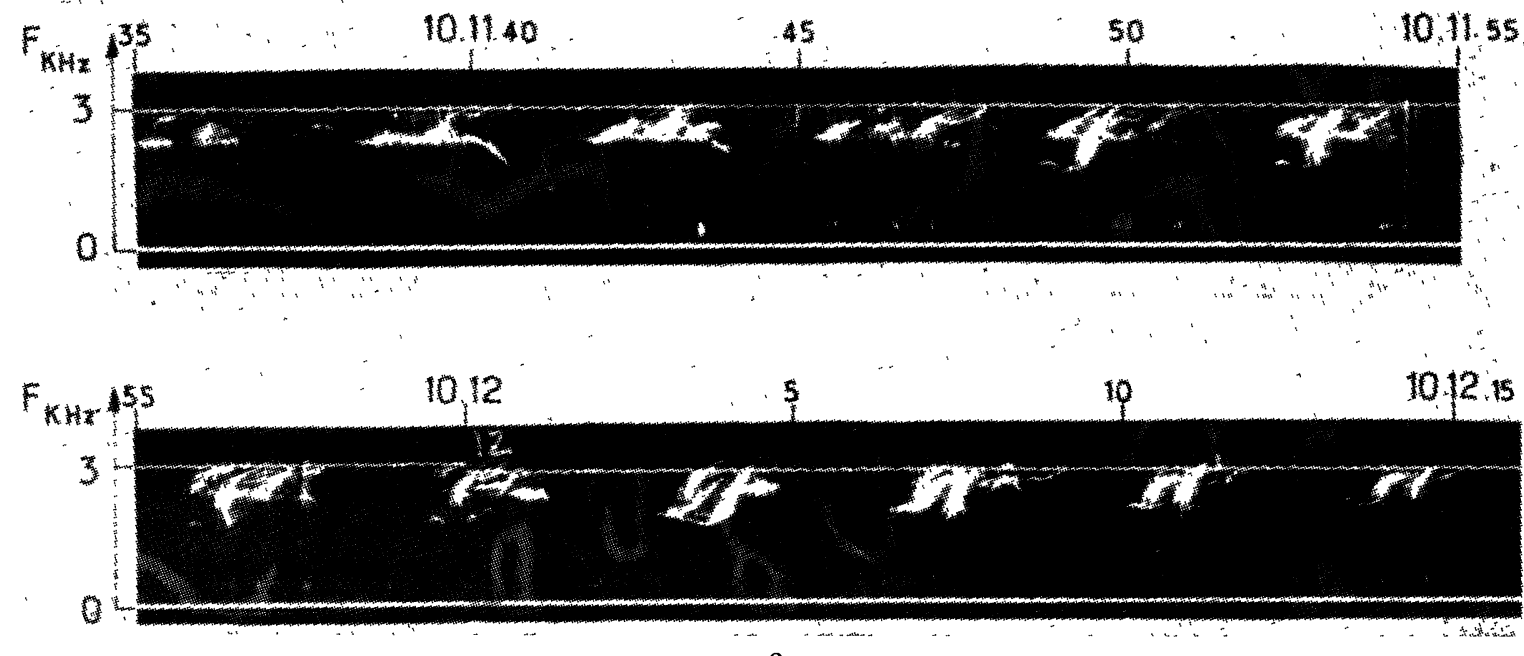

FIG. 8. - Différents types d'émissions T.B.F. :

a) Souffle (GALLET, Proc. I.R.E., 1959, 47); b) Chœur de l'aube (HELIIWELL, op. cit., 1965) ; c) Émissions discrètes (communiqué par Vigneron).

Intensité des bruits dans la magnétosphère et dans le vent solaire. - Les figures 9 et 10 donnent une idée de l'intensité des différents bruits observés dans le voisinage de la Terre (sur la figure 9, la correspondance entre $\gamma \cdot \mathrm{Hz}^{-1 / 2}$ et $\mathrm{W} \cdot \mathrm{m}^{-2} \cdot \mathrm{Hz}^{-1}$ est donnée, dans l'hypothèse où l'indice de réfraction est égal à 1 ). La décroissance de ces bruits en fonction de la fréquence suit à peu près la même loi, que l'on soit dans le vent solaire, dans la magnétogaine ou dans la magnétosphère. Ceci explique pourquoi il est difficile d'enregistrer des phénomènes dans une large bande de fréquence avec un appareil unique. En plus, pour chaque fréquence donnée, l'intensité des bruits peut varier dans de larges proportions, en fonction de l'activité géomagnétique ou solaire. Lorsqu'on a besoin de se fixer des ordres de grandeur pour les émissions discrètes, on peut prendre $\sim 10 \mathrm{~m} \gamma$ pour $1 \mathrm{kHz}$ et $\sim 1 \gamma$ pour $1 \mathrm{~Hz}$ à une valeur de $L=4-5$ dans le plan équatorial.

A noter tout particulièrement la chute rapide du bruit dans le vent solaire dès que la fréquence devient supérieure à la gyrofréquence des protons (fig. $10 \mathrm{c}$ ).
Appareils. - Avant l'apparition des magnétomètres à résonance ou à précession, il était inconcevable d'utiliser des aimants pour détecter les fluctuations rapides du champ magnétique (par rapide, nous entendons les périodes plus courtes que la dizaine de secondes). La sensibilité exigée n'aurait pu être atteinte qu'au prix d'une augmentation de poids préjudiciable à la réponse en fréquence. C'est pourquoi l'utilisation de la loi de Lenz a été d'abord préférée dans ces domaines de fréquence. Un grand nombre de spires bobinées sur un noyau en $\mu$-métal associé à un galvanomètre permettait d'assurer à la fois une bonne sensibilité et une large bande passante (de 500 à 5 s par exemple). Vers les « hautes » fréquences, l'inertie du galvanomètre jouait le rôle d'intégrateur et l'on arrivait ainsi à une bande relativement plate, moyennant une condition simple à réaliser sur l'amortissement global de l'ensemble. C'est le principe des barres-fluxmètres de M. Selzer (fig. 11 a).

Pour les fréquences plus élevées, non seulement l'inertie du galvanomètre devient une gêne, mais la self de la sonde commence à empêcher les tensions 


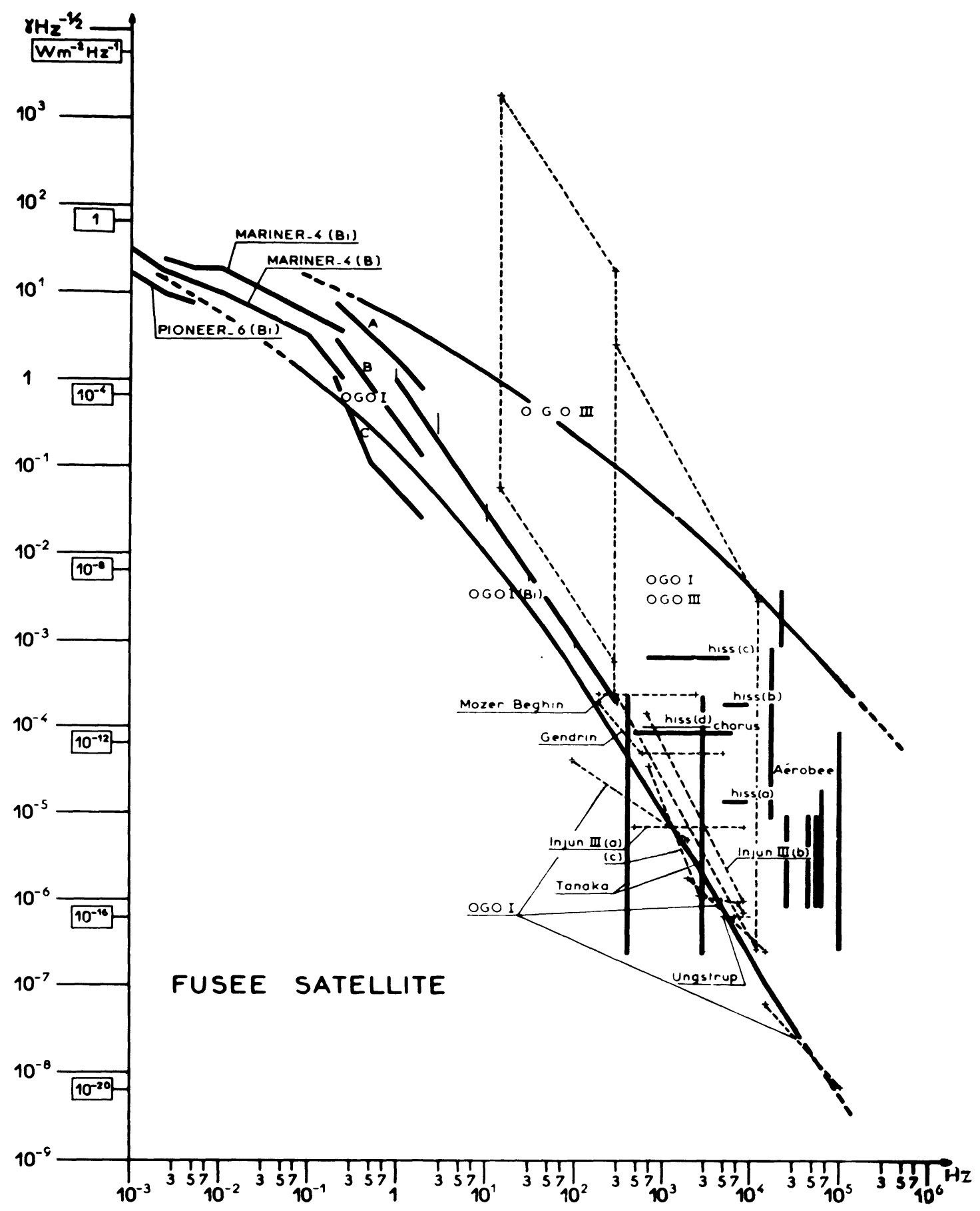

FIG. 9. - Intensités minimales et maximales des bruits U.B.F. et T.B.F. observés en satellite ou en fusée (KARCZEWSKI et VIGNERON, Rapport GRI, nº 23, 1968).

induites de développer les courants correspondants; de plus, les courants de Foucault induits dans la barre constituent une gêne supplémentaire. Une solution a été trouvée par M. Stéfant ( fig. $10 \mathrm{~b}$ ). Elle consiste à utiliser un noyau de ferrite (suppression des courants de Foucault), un bobinage moins important (diminution de la self) et un amplificateur galvanométrique à photocellule (faible bruit) suivi d'une contre-réaction capacitive (élargissement de la bande). Une amélioration a été apportée par MM. Clerc et Gilbert qui ont introduit une contre-réaction de flux, au lieu d une contre-réaction capacitive. La stabilité de gain (rapport de la tension de sortie au champ à l'entrée) est augmentée puisque la contre-réaction agit dès la première fonction de transfert (tension aux bornes de la bobine en fonction du champ magnétique imposé). Le remplacement des galvanomètres par des amplificateurs opérationnels apporte encore à l'heure actuelle une perte de sensibilité, à cause de l'augmentation du bruit des transistors dans les basses fréquences. Le galvanomètre est encore utilisé à cause de son faible facteur de bruit.

Dans les plus hautes fréquences, on peut utiliser des cadres de grande surface sans noyau et à faible nombre de tours, associés à un transformateur-élévateur et un préamplificateur usuel. On peut aussi utiliser le même système que précédemment (fig. $11 \mathrm{c}$ ), sans le galvanomètre, les transistors à effet de champ 


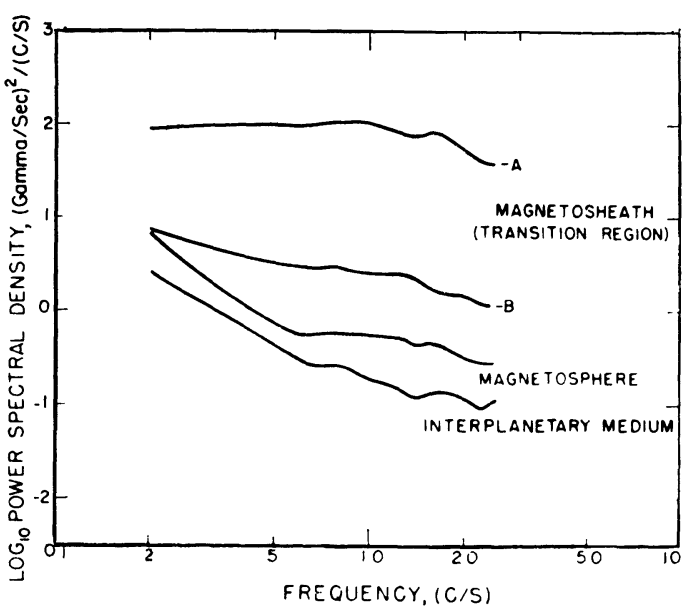

a

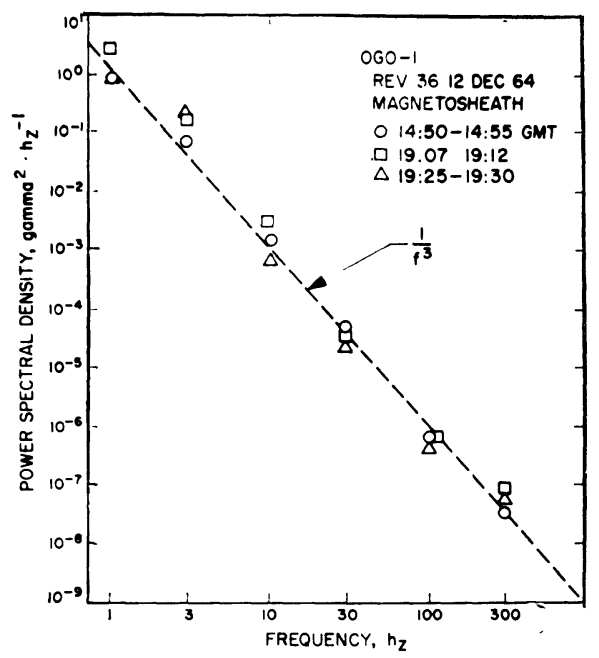

b

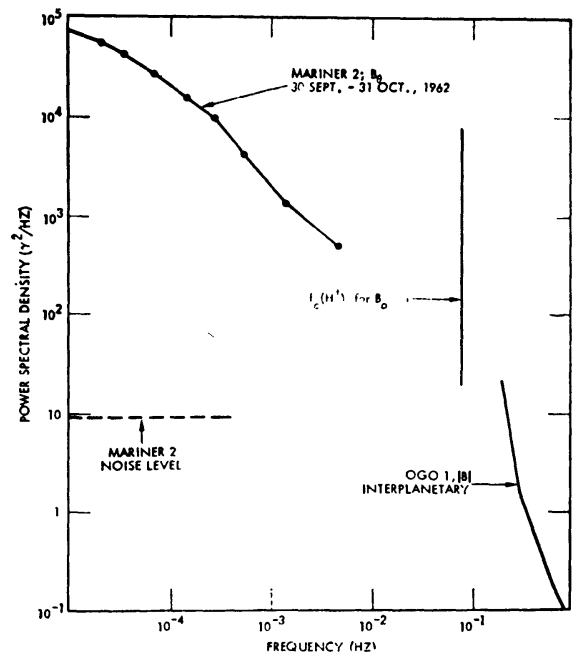

C

FIG. 10. - Intensité moyenne des bruits U.B.F. dans l'espace :

a) Bruit U.B.F. (HOLZER et al., J.G.R., 1966, 71, 1481).

b) Bruit T.B.F. dans la magnétogaine (SMITH et al., J.G.R., 1967, 72, 4803).

c) Bruit dans le vent solaire (KENNEL et SCARF, J.G.R., 1968, 73, 6149).

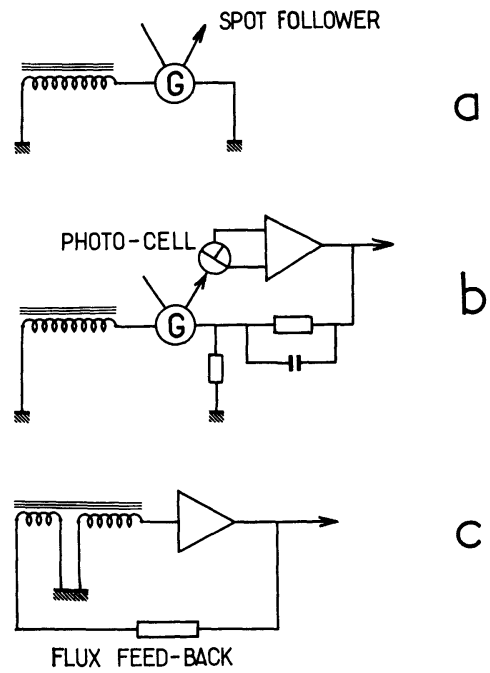

Fig. 11. - Principe des appareils détecteurs employés dans les différentes gammes de fréquence.

(F.E.T.) présentant un facteur de bruit négligeable même aux fréquences inférieures à $200 \mathrm{~Hz}$. Il y a toutefois un compromis à établir, le facteur de bruit n'étant optimal que si la résistance d'entrée du transistor a une valeur bien déterminée, valeur qui dépend de la fréquence.
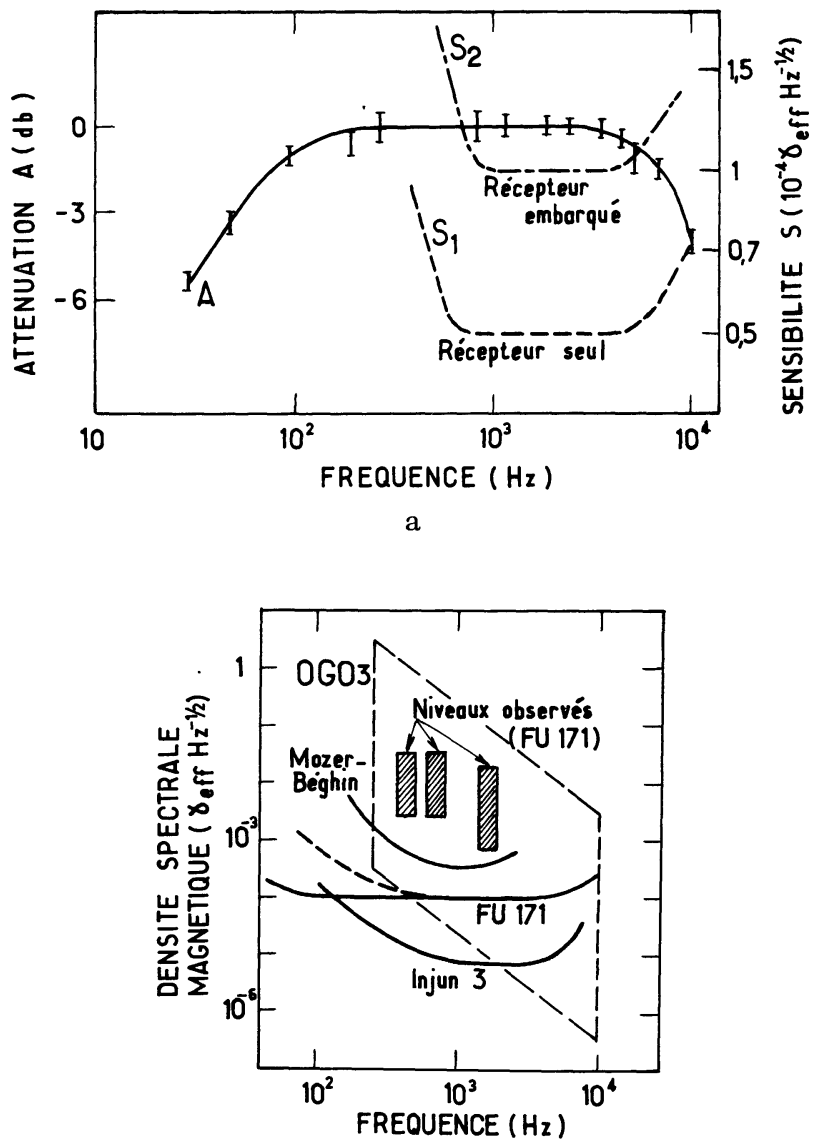

Fig. 12. - Réponse en fréquence et sensibilité des différents capteurs utilisés en fusée ou en satellite :

a) Sensibilité d'une antenne fusée (MEYER, Thèse, 1969)

b) Comparaison de sensibilités (MEYER, Thèse, 1969). 
Les deux systèmes, à boucle et à noyau contreréactionné, ont été utilisés tant au sol qu'à bord d'engins. Le second présente l'avantage d'un volume réduit et des possibilités de calibration en vol. Un exemple des sensibilités ainsi obtenues est donné sur la figure 12 . On notera sur la figure $12 \mathrm{~b}$ que les auteurs ne donnent pas toujours leur sensibilité effective vers les basses fréquences (courbe en traits pointillés) car ils ne tiennent pas compte de l'augmentation du bruit lorsque la fréquence décroît.

Avec l'avènement des nouveaux magnétomètres (à supraconducteurs, à précession ou à pompage optique), il n'est pas exclu de prévoir une extension des bandes passantes vers le haut avec un appareil senseur unique $(0-1 \mathrm{kHz}$ ou plus), sous réserve que ces appareils possèdent la dynamique importante qui est imposée par l'amplitude des phénomènes naturels.

Problèmes posés par l'utilisation des magnétomètres alternatifs dans l'espace. - On connaît, par les exposés de MM. Vuye et Iufer, l'importance des problèmes liés à la propreté magnétique d'un engin sur lequel on veut mesurer des champs magnétiques continus et faibles. D'autres effets interviennent lorsqu'on veut étudier des champs alternatifs et ils ont, jusqu'à un passé très récent, empêché la détection directe des champs U.B.F. dans l'espace. Ces effets sont au nombre de trois.

LA dynamique des PhÉNomènes. - Pour un appareil couvrant simplement deux décades $(0,1 \mathrm{~Hz}$ à $10 \mathrm{~Hz}$ ou $100 \mathrm{~Hz}$ à $10 \mathrm{kHz}$ ), ce qui est peu, la dynamique en champ de l'appareil doit être élevée, d'abord à cause de la loi en $1 / f^{3 / 2}(60 \mathrm{~dB})$, ensuite à cause de la grande variabilité des phénomènes (30 à $40 \mathrm{~dB}$ ), ce qui porte la dynamique totale exigée au voisinage de 90 à $100 \mathrm{~dB}$. Si l'on s'attache à avoir une bande passante plate, ce qui facilite la transmission des formes d'ondes et l'analyse spectrale ultérieure, cette dynamique se retrouve intégralement en sortie de préamplificateur. Pour éviter les phénomènes de saturation, il est donc nécessaire d'assurer une grande dynamique à l'appareil détecteur. Une telle dynamique dans de telles bandes passantes ne pouvant être transmise intégralement pour des questions de télémesure, les différents expérimentateurs se sont orientés vers des systèmes de filtrage et intégration (Injun-3, OGO-1, OGO-3...), de contrôle automatique de gain (Injun-3) ou d'amplificateurs à commutation automatique de gain (FR-1).

Le déplagement du SATEllite. - Bien que toutes les sondes utilisées pour détecter les ondes U.B.F. ne répondent pas aux variations extrêmement lentes, lorsque le satellite passe rapidement dans une région à fort gradient de champ continu, une force électromotrice induite apparaît, dont l'amplitude peut être supérieure à celle des signaux étudiés et saturer l'équipement. C'est le cas pour les satellites terrestres polaires ( 0,3 gauss en $20 \mathrm{mn}$ ) ou pour les sondes destinées à l'étude de Jupiter ( 3 gauss par heure). Si l'on prend comme exemple une sonde dont la réponse est de $10 \mu \mathrm{V}(\gamma . \mathrm{Hz})^{-1}$ associée à un préamplificateur dont le bruit est de l'ordre de $0,1 \mu \mathrm{V}$ (ce qui correspond à une sensibilité de $10 \mathrm{~m} \gamma$ à $1 \mathrm{~Hz}$ ), on voit que ces champs perturbateurs correspondent à des tensions de l'ordre de $500 \mu \mathrm{V}$, c'est-à-dire à $70 \mathrm{~dB}$ au-dessus du bruit. Un filtrage supplémentaire, difficile à réali- ser de façon stable dans ces basses fréquences, ne réduirait ce chiffre que de 30 à $40 \mathrm{~dB}$ si l'on veut conserver une réponse plate au-dessus de $0,1 \mathrm{~Hz}$.

LA RotATION DE L'ENGIN SUR LUI-MÊME. — C'est le problème le plus délicat. Il est évident que si le satellite se trouve dans une configuration telle que l'axe de détection du magnétomètre tourne dans un plan qui contient la direction du champ magnétique continu $B_{0}$, il sera impossible de mesurer des perturbations d'amplitude $\mathrm{b}$ du champ aux pulsations $\omega$ voisines de la pulsation de rotation $\Omega$ de l'engin. Prenons comme exemple un satellite stabilisé par spin à 1 tour par $\mathrm{s}$ et situé à une altitude relativement basse $(1000 \mathrm{~km})$, on a $B_{0} \sim 20000 \mathrm{\gamma}, \Omega \sim 6$.

Le signal induit dans la sonde par la rotation $\mathrm{du}$ capteur dans le champ continu $B_{0}$ sera $2 \times 10^{6}$ fois plus fort que les signaux de l'ordre de $10 \mathrm{~m} \gamma$ que l'on cherche à détecter. Les moyens qui permettent de pallier cet effet parasite sont nombreux; à l'exception du dernier, nous verrons qu'ils réduisent considérablement les possibilités de mesurer dans l'espace proche des champs électromagnétiques U.B.F. (dans l'espace lointain, les problèmes sont différents car $B_{0}$ ne vaut que $\sim 10 \gamma$ et l'on peut éliminer à l'analyse le signal parasite, $10^{3}$ fois plus fort, qui n'a pas provoqué de saturation puisque nous avons vu que la dynamique est au moins de $80 \mathrm{~dB}$ ).

Premier palliatif. - Rejeter la fréquence de rotation de l'engin en dehors de la bande passante d'analyse. Si l'on ne s'intéresse qu'aux ondes T.B.F. $(f>300 \mathrm{~Hz})$, un filtrage sérieux permet d'éliminer cet effet parasite. On peut aussi essayer de limiter la vitesse de rotation de l'engin ( 1 tour en 10 ou $20 \mathrm{~s}$ est courant pour les satellites d'un certain poids), ce qui permet d'abaisser la fréquence utile d'analyse.

Deuxième palliatif. - Utiliser une configuration dans laquelle le mouvement du satellite dans le champ magnétique directeur $B_{0}$ n'induit pas d'effets parasites. Ceci peut être obtenu de deux façons. Soit en se limitant à mesurer une composante parallèle à l'axe de spin; le seul effet parasite que l'on observera sera dû au mouvement de précession, mais ce mouvement est à une fréquence beaucoup plus basse et souvent d'amplitude très faible (pour les satellites tout au moins; il n'en est pas de même pour les fusées). Soit en mesurant des composantes perpendiculaires à l'axe de spin, mais en s'arrangeant pour que celui-ci soit plus ou moins parallèle au champ magnétique directeur. Une telle configuration est envisageable pour les satellites équatoriaux pour lesquels on peut choisir un axe de spin parallèle à l'axe de la Terre et garder un parallélisme approché avec $B_{0}$. Même dans ce cas, comme la direction de $B_{0}$ n'est pas exactement perpendiculaire à l'équateur géographique, il faut se limiter aux grandes distances géocentriques $\left(5-6 R_{T}\right)$ pour lesquelles $\left|B_{0}\right|$ reste faible.

Troisième palliatif. - Il consiste à supprimer l'effet parasite avant la transmission par la télémesure en réinjectant un signal à la fréquence de rotation. Le principe, étudié par le Docteur Sonnett aux ÉtatsUnis, en est le suivant.

A partir d'un senseur solaire, on peut reconstituer avec suffisamment de précision (par suite de la stabilité du mouvement) une sinusoïde dont la fréquence 
est égale à la fréquence de rotation de l'engin. Si $H_{x}$ et $H_{y}$ sont deux composantes mesurées dans un plan perpendiculaire à l'axe de spin, et si $H_{0}$ est la composante continue du champ, et $h$ l'amplitude de l'oscillation, à la pulsation $\omega$, que l'on cherche à détecter, les combinaisons :

$$
\begin{aligned}
& H_{x} \sin \Omega t+H_{y} \cos \Omega t \\
& \text { et } \quad H_{x} \sin \Omega t-H_{y} \cos \Omega t
\end{aligned}
$$

permettent d'obtenir d'une part le signal $H_{0}$ modulé avec la pulsation $2 \Omega$, d'autre part le signal cherché $h \sin \omega t$. Il y a bien entendu des effets de modulation parasites et d'harmoniques, qui dépendent de l'orientation respective des vecteurs, $H_{0}, h$ et de l'axe de spin. Une telle méthode a néanmoins fonctionné; elle a permis d'étendre la gamme de fonctionnement des magnétomètres continus au-delà de la fréquence de rotation de l'engin.

Conclusion. - A la lecture de ces différentes remarques, il semblerait que l'on puisse dresser les conclusions suivantes :

- En ce qui concerne la mesure des champs magnétiques continus, les problèmes posés par la propreté magnétique des engins spatiaux sont tels qu'ils rendent inutile pour l'instant l'utilisation spatiale de magnétomètres ayant une sensibliité meilleure que $10^{-6}-10^{-7}$ gauss.

- Pour les champs alternatifs $(f>0,2 \mathrm{~Hz})$, les bobines à induction (« search coils ») remplissent pour l'instant les conditions de sensibilité et de dynamique requises. Par ailleurs, les effets parasites dus au déplacement et à la rotation de l'engin dans le champ magnétique directeur sont d'un ordre tel qu'il est illu- soire d'essayer d'améliorer de beaucoup la sensibilité des détecteurs dans ce domaine de fréquence, et qu'il importe plutôt de trouver les « astuces » électroniques qui permettent de s'affranchir de ces effets.

Une telle conclusion « conservatrice », si elle est probablement juste dans le domaine des recherches sur l'environnement proche de la Terre, ne l'est certainement pas relativement aux problèmes posés par la structure du vent solaire, principalement aux grandes distances héliocentriques.

En effet, la nature turbulente de ce milieu, les discontinuités qu'il présente, sont d'un intérêt fondamental pour la compréhension d'un bon nombre de problèmes d'astrophysique. Compte tenu de la faible valeur des champs $(\sim 1 \gamma$ aux environs de l'orbite de la planète Jupiter), les mesures continues doivent être étendues à des valeurs plus faibles, avec une précision absolue certaine (on sait que des conclusions erronées, relatives à la structure du vent solaire avaient été obtenues par suite d'une erreur systématique absolue de l'ordre de $2 \gamma$ dans les mesures faites avec des magnétomètres à résonance). Il y a donc là un progrès à faire. La sensibilité relative doit également être accrue si l'on veut détecter les ondes hydromagnétiques qui se propagent dans le vent solaire; il est d'ailleurs évident qu'il n'y a plus lieu de séparer la partie continue des parties U.B.F. et T.B.F., pourvu que la dynamique du détecteur le permette. Il n'est donc pas impensable de dire que des sensibilités de l'ordre de $10^{-9}$ - $_{-}$ $10^{-10}$ gauss avec des bandes passantes pouvant s'étendre jusqu'à la centaine de hertz (la gyrofréquence électronique dans le vent solaire) forment un objectif valable pour la mesure dans l'espace des champs magnétiques faibles, pour les dix prochaines années.

\section{BIBLIOGRAPHIE}

On pourra consulter les quatre travaux généraux suivants, à partir desquels d'autres références pourront être trouvées facilement :

KING (J. W.) et Newman (W. S.), "Solar-Terrestrial Physics ", Academic Press, New York, 1967.

GENDRIN (R.), Progrès récents dans l'étude des ondes T.B.F. et E.B.F., Space Sci. Rev., 1967, 17, 314-395.
HELIIWELI, (R. A.), "Whistlers and related ionospheric phenomena ", Stanford University Press, Stanford, 1965.

Troitskaya (V. A.) et Gul'Elmi (A. V.), Geomagnetic micropulsations and diagnostics of the magnetosphere, Space Sci. Rev., 1967, 5/6, 689-768. 